\title{
Sensitivity of the General Linear Model to Assumptions
}

\author{
Mahsa Jabbar, Hamidreza Ghorbaniparvar, Fatemeh Ghorbaniparvar
}

\begin{abstract}
Non-Gaussian noise often causes in significant performance abatement for systems which are designed using Gaussian assumption. This report challenges the question of General Linear Model with White Gaussian Noise assumption in order to define the sensitivity of the performance of an optimal estimator. Gaussian noise models provide an important role in many signal processing applications. The Laplacian and Uniform signal are two worthy examples of noise that can be compared to the White Gaussian Noise, though the sensitivity which can be compared with any non-Gaussian. White Gaussian Noise has been considered for General Linear Models and deviation from whiteness would affect on our estimates under different circumstances. Moreover, new assumptions have been considered to generate different type of signals in order to evaluate the sensitivity of the General Linear Model.

Keywords: Non-Gaussian noise, optimal estimator, Laplacian signal, Uniform Signal, Gaussian liner model.
\end{abstract}

\section{INTRODUCTION}

The General Linear Model (GLM) is a common conditional probability model where it can be utilized for a variety of statistics models and contains an absorbing computational property. It is also a key tool for the optimal estimators due to the distinguished properties that the model contains. The majority of the estimator always covers an assumption of Gaussian signals due to Central Limit Theorem (CLT).

The GLM sensitivity is assumed under two conditions where the first condition takes on a diverse probability models and the second condition investigates the model assuming the known and unknown correlation between samples. Moreover, a statistical property of WGN is compared with these assumptions.

Primarily, a probability model in the GLM has been derived. An approach to determine the sensitivity of a GLM assumes Gaussian Model and Non-Gaussian Model. The signals processed are: Gaussian-Gaussian Mixture (GMM), Guassian-Uniform Mixture (GUM), Laplacian, Weibull, and Uniform. Non-Gaussian noise is used to address and compare the observation of the noises based on an estimation experiment in [1].

The optimal estimator for the GLM recognizes a known

Revised Manuscript Received on March 05, 2020.

* Correspondence Author

M. Jabbar* Department of Chemistry at University at Buffalo, Buffalo, NY 14260 , USA.

H. Ghorbaniparvar, University at Buffalo, Buffalo, NY 14260, USA.

F. Ghorbaniparvar, Electrical Engineering Department at Iran University of Science and Technology Tehran, Iran.

(c) The Authors. Published by Blue Eyes Intelligence Engineering and Sciences Publication (BEIESP). This is an open access article under the CC BY-NC-ND license (http://creativecommons.org/licenses/by-nc-nd/4.0/) covariance and theoretically we assume that the signal is occasionally white. Our goal is to understand the sensitivity of optimal estimator performance to the deviation from the white noise.

The goal of the project is evaluating the sensitivity of GLM under the circumstances. In this paper we will discuss our simulation results based on GLM with its assumptions. Section II will be a review of literature about optimal estimators and Section III will propose a methodology to construct the models. The next two sections (Section IV and V) will provide the insights of GLM assumptions. Finally, the report will conclude our research in Section VI.

\section{REVIEW OF LITERATURE}

Minimum Variance Unbiased (MVU) and Maximum Likelihood (ML) estimators are usually difficult to determine without being represented by a general linear model[1]-[3]. [4] emphasizes the optimal estimators in linear model allows everyone to analyze the problem within the model due to its unique properties. The idea of having optimal estimators is due to the small variance values within their estimator. ML estimators for instances, are considered than other types of estimates because of its small variance being distributed closer to the value.

As referenced from the works of [1], the bulk of the research in Non-Gaussian noise is limited to independent and identical distributed observation sequences due to the difficulty in characterizing correlated multidimensional Non-Gaussian PDFs. The probability density function (PDF) of the Gaussian states:

$$
\begin{aligned}
& p(x ; \theta)=\frac{1}{(\theta-1) / 2} \exp \left[-\frac{1}{2}(x-\right. \\
& \left.\mu(\theta))^{T} C^{-1}(\theta)(x-\mu(\theta))\right]
\end{aligned}
$$

Where $\boldsymbol{x}$ states our sample, the covariance matrix is $\boldsymbol{C}$, and mean is $\boldsymbol{\mu}$. As it can be seen throughout the literature in [11]-[18], white Gaussian noise assumption is a widely used assumption. So, it is important to investigate the effects of different noises.

\section{METHODOLOGY}

The GLM assumptions permit a certain estimator in the investigation of the signal.

General Linear Model assumes White Gaussian Noise which plays significant role on the estimation. There are suitable optimal estimators for deriving the sensitivity of the GLM. Consider our model with the Gaussian assumption:

$$
\boldsymbol{x}=\boldsymbol{H} \boldsymbol{\theta}+\mathrm{w}
$$

where

\section{Published By:}

Blue Eyes Intelligence Engineering \& Sciences Publication (C) Copyright: All rights reserved. 


$$
\begin{aligned}
& \boldsymbol{x}=[x[0] x[1] \ldots x[N-1]]^{Y} \\
& \boldsymbol{w}=[w[0] w[1] \ldots w[N-1]]^{Y} \\
& \boldsymbol{\theta}=[A B]^{T} \\
& \text { and }
\end{aligned}
$$$$
H=\left[\begin{array}{cc}
1 & 0 \\
1 & 1 \\
\vdots & \vdots \\
1 & N-1
\end{array}\right]
$$

The matrix $\boldsymbol{H}$ is the observation matrix with data $\boldsymbol{x}$ being observed after the estimator $\boldsymbol{\theta}$ is operated by the matrix. $\theta=[A B]^{T}$ is the parameter vector. The noise vector $\boldsymbol{w}$ is usually assumed Gaussian according to [4]. The GLM can be also represented as an observation data:

$$
x[n]=A+B n+w[n] \text { where } n=0,1_{s \ldots}, N-1
$$

$w[n]$ is commonly considered as the WGN and $A \& B$ are our unknown parameters which will be selected to assess the estimator's sensitivity.

Our noise $\boldsymbol{w}$ is modeled zero mean with different probability density functions. Optimal estimator would be used to assess the sensitivity of model to assumptions. Based on the Table I, that is the optimal estimator for the model due to the fact that if the noise is WGN we would have MVU estimator and in worst case scenario where the probability model is unknown, it would be a Least-Square Estimator [11]. We derived our own noise model as shown in eq. (4).

$$
p\left(x ; \sigma^{2}\right)=(1-\alpha) * p\left(x ; \sigma^{2}\right)+\alpha * p\left(x ; \sigma^{2}\right)
$$

Eq. 4 is used as the building block in creating the mixture models of the GMM and GUM. Laplacian, Weibull and Uniform noise separately applied to the model and compared with the case where noise is White Gaussian.

Another assumption is considered White Gaussian Noise when the Colored Gaussian Noise is constructed to investigate the sensitivity of the model to deviation from whiteness. Moreover, a suitable approach is to create various Color Gaussian Noises and compare to the White Gaussian Noise.

$$
\boldsymbol{C}=\left[\begin{array}{cccc}
\sigma^{2} & \sigma^{2} \mathrm{~B} & \cdots & \sigma^{2} \mathrm{~B}^{N-1} \\
\sigma^{2} \mathrm{~B} & \cdots & \sigma^{2} \mathrm{~B} & \vdots \\
\vdots & \sigma^{2} \mathrm{~B} & \sigma^{2} & \sigma^{2} \mathrm{~B} \\
\sigma^{2} \mathrm{~B}^{N-1} & \cdots & \sigma^{2} \mathrm{~B} & \sigma^{2}
\end{array}\right]
$$

The covariance matrix listed in eq. (5) constructed to assess the sensitivity of the performance of estimator to deviation from whiteness, thus B authorizes us to manage the power of correlation between the samples in order to see its

\begin{tabular}{|c|c|c|}
\hline Model & Parameters & Estimate \\
\hline $\mathrm{x}=\mathrm{H} \theta+\mathrm{e}$ & $\begin{array}{l}\text { No Probability } \\
\text { Model Needed }\end{array}$ & $\widehat{\theta}_{\mathrm{LS}}=\left(\mathrm{H}^{\mathrm{T}} \mathrm{H}\right)^{-1} \mathrm{H}^{\mathrm{T}} \mathbf{x}$ \\
\hline $\mathrm{x}=\mathrm{H} \theta+\mathrm{w}$ & $\begin{array}{l}\text { PDF Unknown, } \\
\text { White Noise }\end{array}$ & $\widehat{\theta}_{\mathrm{BLUE}}=\left(\mathrm{H}^{\mathrm{T}} \mathrm{H}\right)^{-1} \mathrm{H}^{\mathrm{T}} \mathbf{x}$ \\
\hline $\mathrm{x}=\mathrm{H} \boldsymbol{\theta}+\mathrm{w}$ & $\begin{array}{l}\text { PDF Gaussian, } \\
\text { White Noise }\end{array}$ & $\widehat{\theta}_{M L}=\left(H^{T} H\right)^{-1} H^{T} \mathbf{x}$ \\
\hline $\mathrm{x}=\mathrm{H} \theta+\mathrm{w}$ & $\begin{array}{l}\text { PDF Gaussian, } \\
\text { White Noise }\end{array}$ & $\widehat{\theta}_{M V U}=\left(H^{\mathrm{T}} \mathbf{H}\right)^{-1} \mathrm{H}^{\mathrm{T}} \mathbf{x}$ \\
\hline
\end{tabular}
effects on estimator variance. The value is set from $\mathbf{0}$ to $\mathbf{1}$ where zero means White Gaussian Noise and a value of one means high correlation between samples. Furthermore, the knowledge of probability model would be explored on estimator performance.

Table I: Comparison of Estimators
A list of models with their parameter conditions stating the appropriate estimator to be used [10]

\section{GENERAL LINEAR MODEL}

\section{A. Assumption}

Our general linear model is based on equations (2) \& (3) based on [4]'s definition of a standard linear model and equations. He reiterates optimal estimators are a difficult task to resolve, but dealing with a GLM representation makes any estimation processing easy to determine. The Gaussian signal is assumed in the GLM, thus the additions of other non-Gaussian model will provide significant data and results.

\section{B. Mixture Models}

As we assumed Gaussian in the GLM, we developed two mixture noise models. The Mean Square Error of estimates illustrated in Fig.1 interprets two unknown variables $A$ and $B$ which is our parameter of interest. Two Gaussian noises are mixed to form a Gaussian-Gaussian Mixture noise, in addition Gaussian-Uniform noise is the second mixture. The Uniform noise isn't a common signal encountered in the world of signal processing but it provides a useful comparison with Gaussian noise because the linear average is comparatively poor estimator for the mean of a uniform distribution [8].

The relation between the Mean Square Error (MSE) and Mixture Weight $(\alpha)$ is dependent of each other. The red line in Fig.1. represents the Gaussian-Gaussian (double-Gaussian) mixture noise. The weight of the mixture when $\alpha=0$ and $\alpha=1.0$ demonstrates that the mixture is single White Gaussian. The MSE decreases when $\alpha$ is going toward 5 from both sides. A double-Gaussian noise mixture model shows a characteristic curve similar to a parabola ' $U$ ' curve.

Uniform noise is mixed with the Gaussian noise forming the blue curved in Fig.1 which is known as the Gaussian-Uniform Mixture model. Unlike the double-Gaussian mixture, the MSE decreases as the weight of the mixture rises. The uniform-Gaussian Mixture Model deviates its path after $\alpha>2$, where the MSE value continues to decrease as the weight of the mixture increases.

A possible perceptive about its small decrease of the MSE happens when any other non-Gaussian noise is combined with Gaussian.

The MSE of the mixture models is focusing on each of the unknown parameters being estimated. In the MSE of parameter A the value is less than the MSE of parameter B.

As illustrated in Fig.1, probability model of noise has significant effect on MSE thus adding noise with similar characteristics to data could be considered a way of improving estimator accuracy though it needs more investigation. 

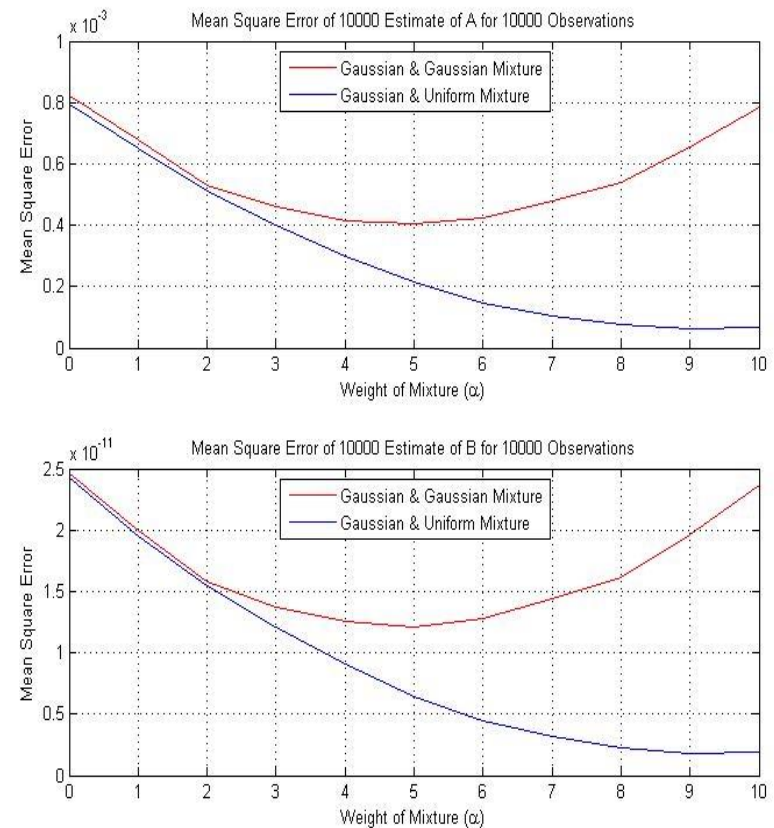

Fig.1. The Mean-Square Error (MSE) displaying the two mixture model ran with 10000 samples and observations for the estimation of parameters $A$ and $B$.

\section{Gaussian vs Non-Gaussian Noise Models}

Based on the observation data of the mixture models, the Gaussian-uniform noise deviates from the double-Gaussian mixture model after the first two weight of mixture. We can determine that our uniform noise would generate a result after using our optimal estimator on all our noises. Based on Fig.2 our Gaussian and non-Gaussian noise model is going through the optimal estimator. Laplacian and Weibull produced a similar result to the Gaussian noise, however uniform noise displays a significant effect on parameter estimation which means the MSE considerably decreased compared to Gaussian noise.

\section{Cramer-Rao Lower Bound (CRLB) Analysis}

Based on our GLM from equation (2) and (3), we considered the line fitting of the model using the observation from equation (3). $w[n]$ is our WGN or white Gaussian noise and determining the CRLB for slope $B$ and intercept $A$ based on [5]'s definition of the GLM's CRLB. For every distribution there is different CRLB, thus derived CRLB just applies to WGN in our model.

Our CRLB is:

$$
\begin{aligned}
& \operatorname{var}(\hat{A}) \geq \frac{2(2 N-1) \sigma^{2}}{N\left(N^{2}-1\right)} \\
& \operatorname{var}(\hat{B}) \geq \frac{12 \sigma^{2}}{N\left[N^{2}-1\right]}
\end{aligned}
$$

Our observation of the CRLB where $A$ has increased over that obtained when $B$ is known. The DC level in WGN contains:

$$
\operatorname{var}(\hat{A}) \geq-\frac{1}{E\left[\frac{\left[^{2} \ln p[x, A]\right.}{\mathbb{E} A^{2}}\right]}=\frac{\sigma^{2}}{N}
$$
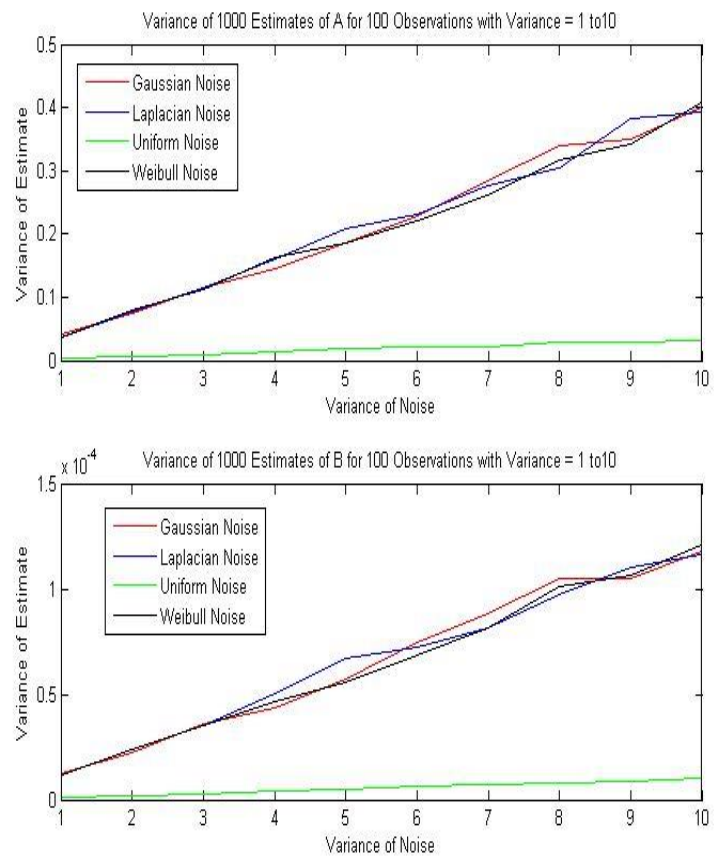

Fig.2. The Gaussian Noise's variance is estimated among with three other non-Gaussian noise: Laplacian, Uniform, and Weibull.

The general result that asserts the CRLB always increases as we estimate more parameters. There is also this point:

$\frac{\operatorname{CRLB}(A)}{\operatorname{CRLB}(B)}=\frac{(2 N-1)(N-1)}{6}>1$

For $N \geq 3, B$ is easier to estimate and its CRLB decreases as $1 / \mathrm{N}^{3}$ as opposed to the $1 / \mathrm{N}$ dependence for the CRLB of $A$. These differing dependences indicate that $x[n]$ is more sensitive to changes in $B$ than to changes in $A$ as indicated in [5-6].

As seen in Fig.3, the estimator for the Gaussian noise lays on the CRLB as the number of samples increases and the same result when shown in Fig.4 where the green line is seen clearly is the CRLB for WGN. So the estimator for the WGN reaches its lower bound which is the CRLB.

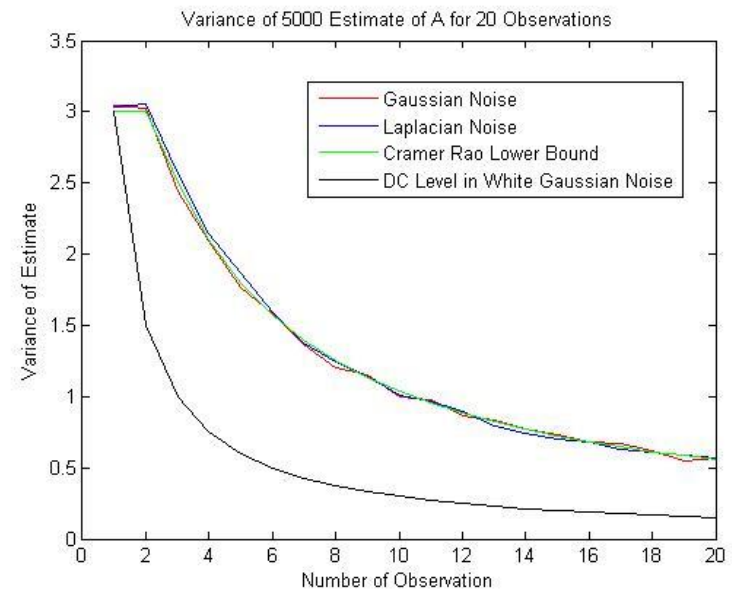

Fig.3. The DC Level in the WGN and where it stands with the Gaussian CRLB and the Gaussian and Laplacian Noise. 


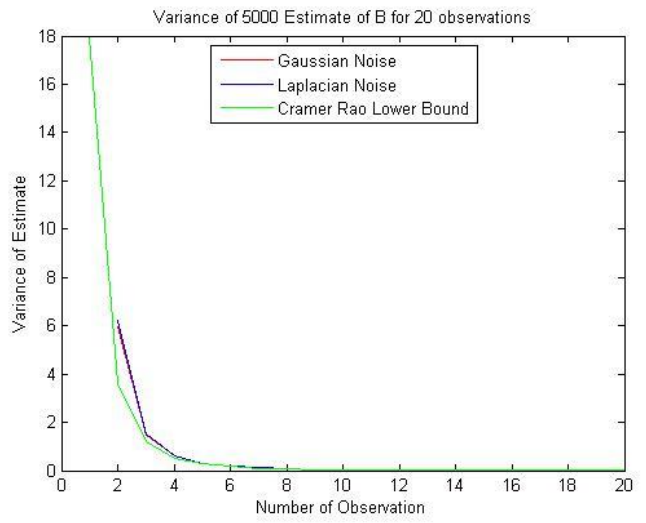

Fig.4. The relation of the Gaussian and Laplacian noise and its CRLB

\section{GENERAL LINEAR MODEL WITH KNOWN COVARIANCE}

\section{A. Assumptions}

Based on the first GLM with the assumption of a Gaussian noise, the second GLM has two assumptions that have been considered. The first case considers the whiteness of the noise to the performance of the optimal estimator on the deviation of the noise correlation. The second case reflects on the covariance matrix where there isn't any known probability model. The model noise has been constructed through the covariance matrix based on equation (4). We are going to estimate the unknown parameters $\boldsymbol{A}$ and $\boldsymbol{B}$ from the model equation (3) when the probability model of noise is known and unknown based on Table I. Eq. (10) shows the effects of the knowledge of probability model [21].

$$
\widehat{\theta}_{M v U}=\left(H^{\mathrm{T}} \mathrm{C}^{-1} \mathrm{H}\right)^{-1} \mathrm{H}^{\mathrm{T}} \mathrm{C}^{-1} \mathbf{x}
$$

\section{B. Deviation from Whiteness}

As Fig.5 illustrates, the White Gaussian Noise (WGN) lays on its CRLB which is designated for the WGN. On the other hand when the noise of our signal model is a Non-White Gaussian Noise (NWGN), the variance of the estimator dramatically increases whether the covariance is known or unknown. In addition, the variance of our estimator would rise even more when there isn't any knowledge in terms of the correlation between the samples. Since the covariance plays a crucial role in the probability model, the correlation between samples seen in Fig.5 would decrease the accuracy of the estimator.

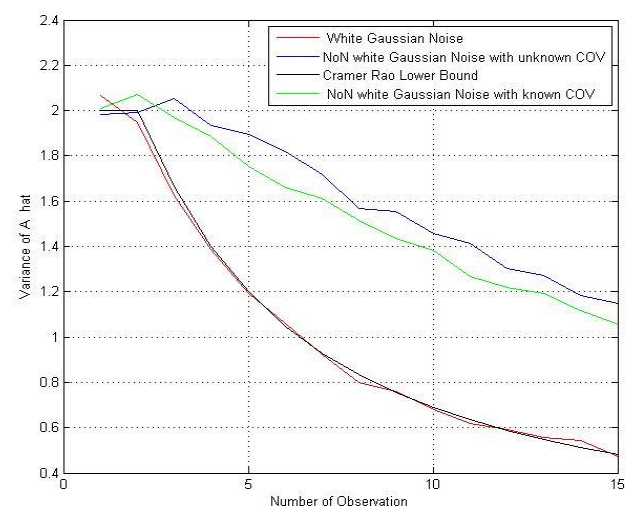

Fig.5. The relationship of the Gaussian Noise when the covariance is known and unknown comparing to the Gaussian laying on its CRLB.
As seen in Fig.6 and Fig.7, we change the variance of B which is used to generate the covariance matrix back in equation (5). The effects of $B$ which forms the covariance matrix will be discussed. Fig. 6 shows when $B=0$, there is no correlation between the samples and the estimator operates to the case when the NWGN effected our model by increasing the $B$. Therefore, the variance of our estimator would increase extremely. In Fig. 6 the relation between the two NWGN displays the NWGN with an unknown covariance matrix having a greater variance of estimates than the NWGN with a pre-assigned covariance and even it would be more when the coefficient of the covariance matrix increases. The same rule applies in Fig.7 when the parameter $\mathrm{B}$ is being estimated.

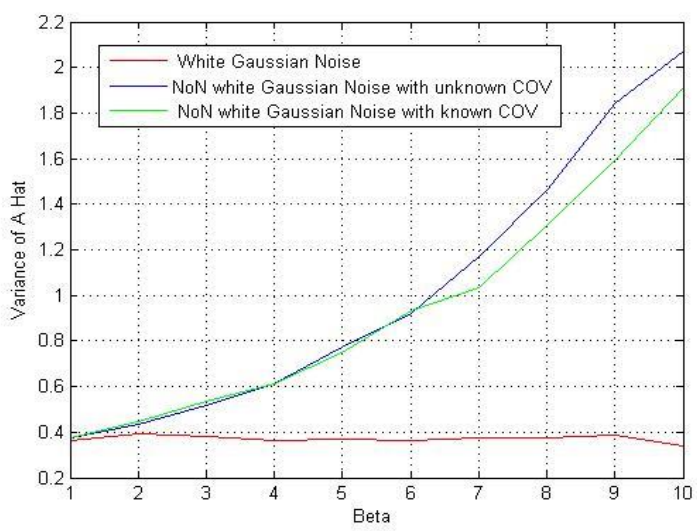

Fig.6. Estimating parameter, A with the relation of the WGN and NGWM

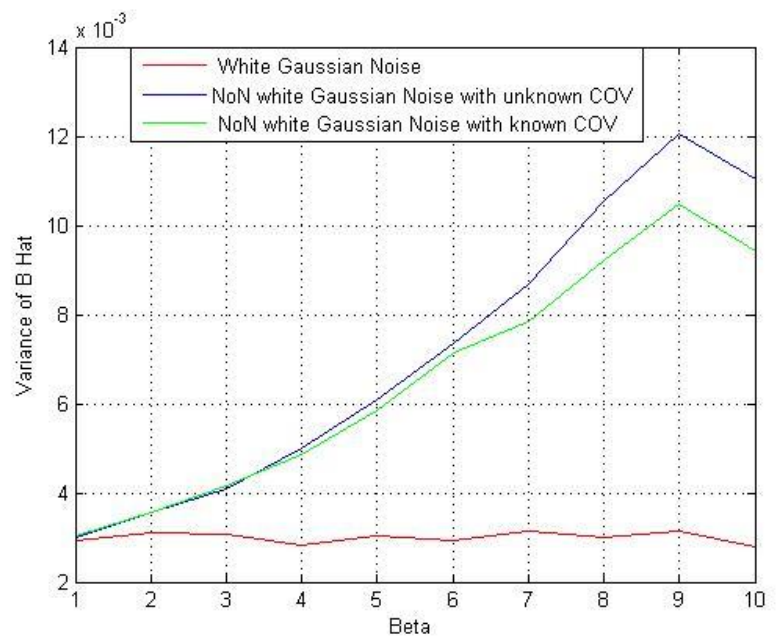

Fig.7. Estimating parameter $B$ with the relation of the WGN and NGWM

\section{CONCLUSION}

Applying different approaches to generate noise, we have explored the sensitivity of the GLM under assumptions. As our simulation indicated in the first GLM assumption, the Laplacian and Weibull signals have a similar behavior with WGN in case of optimal estimators.

Published By:

Blue Eyes Intelligence Engineering \& Sciences Publication (C) Copyright: All rights reserved. 
Moreover, the correlation between the samples increases the variance of the estimator. In future works, the color noises with different power spectrum can be applied where the model assumes WGN in order to deeply investigate the sensitivity of the optimal estimator.

\section{REFERENCES}

1. Pradeepa and G.V. Anand, "Estimation of Signals in Colored Non Gaussian Noise Based on Gaussian Mixture Models," Dept. Elect. Comm. Eng., Indian Instit. Of Sci., Bangalore- 560 012, India, Rep 1-4244-0581-5/06

2. Abdoli M.A., Golzary A., Hosseini A., Sadeghi P. Wood Pellet as a Renewable Source of Energy. University of Tehran Science and Humanities Series. Springer, Cham, 2018.

3. H. Sangrody, N. Zhou, and X. Qiao, "Probabilistic models for daily peak loads at distribution feeders," in Power \& Energy Society General Meeting, 2017 IEEE, 2017, pp. 1-5.

4. S.M. Kay, "Fundamentals of Statistical Signal Processing, Volume I: Estimation Theory," 1st ed.. Upper Saddle River, NJ: Prentice Hall PTR, 1993, pp. 83-84.

5. L.R. Shenton and K.O. Bowman, "Maximum likelihood estimation in small samples," Monograph No. 38. New York, NY: Macmillan Publishing Co., Inc, 1977, pp. 1-8.

6. S.M. Kay, "Fundamentals of Statistical Signal Processing, Volume I: Estimation Theory ," 1st ed.. Upper Saddle River, NJ: Prentice Hall PTR, 1993, pp. 15-22, 157.

7. Abdoli M.A., Golzary A., Hosseini A., Sadeghi P. Wood Pellet Emissions. In: Wood Pellet as a Renewable Source of Energy. University of Tehran Science and Humanities Series. Springer, Cham, 2018.

8. T. Veldhuizen. (1998, January 16). Noise Model (1st Ed.) [Online]. Available: http://homepages.inf.ed.ac.uk/rbf/CVonline/LOCAL_COPIES/VELDH UIZEN/node11.html

9. Abdoli M.A., Golzary A., Hosseini A., Sadeghi P. Wood Pellet Combustion. In: Wood Pellet as a Renewable Source of Energy. University of Tehran Science and Humanities Series. Springer, Cham, 2018

10. S.M. Kay, "Fundamentals of Statistical Signal Processing, Volume I: Estimation Theory ," 1st ed.. Upper Saddle River, NJ: Prentice Hall PTR, 1993, pp. 41-43.

11. M. Fowler. EECE 522. Class Lecture, Topic: "Chapter 8 Least Squares Estimation." Thomas J. Watson School of Electrical and Computer Engineering and Applied Science, Binghamton University, Binghamton, NY, Mar. 18, 2014.

12. M. Ghorbaniparvar, "Survey on forced oscillations in power system." Journal of Modern Power Systems and Clean Energy 5, no. 5 (2017): 671-682.

13. Ghorbaniparvar, Mohammadreza, Xiaohua Li, and Ning Zhou. "Demand side management with a human behavior model for energy cost optimization in smart grids." In Signal and Information Processing (GlobalSIP), 2015 IEEE Global Conference on, pp. 503-507. IEEE, 2015

14. Abdoli M.A., Golzary A., Hosseini A., Sadeghi P. Biomass as a Source of Energy. In: Wood Pellet as a Renewable Source of Energy. University of Tehran Science and Humanities Series. Springer, Cham, 2018.

15. M. Ghorbaniparvar., and N. Zhou. "Bootstrap-based hypothesis test for detecting sustained oscillations." In Power \& Energy Society General Meeting, 2015 IEEE, pp. 1-5. IEEE, 2015.

16. M. Jabbar, and H. Ghorbaniparvar. "Use of GC-MS combined with resolution methods to characterize and to compare the essential oil components of green and bleached cardamom." IJRCE 5 (2014).

17. Abdoli M.A., Golzary A., Hosseini A., Sadeghi P. Wood Pellet Production Standards, In: Wood Pellet as a Renewable Source of Energy. University of Tehran Science and Humanities Series. Springer, Cham, 2018.

18. M. Jabbar, and H. Ghorbaniparvar. "Determination of Volatile Components in Black Cardamom with Gas Chromatography-Mass Spectrometry and Chemometric Resolution ." International Journal of Engineering Research and Technology 3 (11), 1280-1286 2014.

19. B. Khorramdel, H. Khorramdel, A. Zare, N. Safari, H. Sangrody, and C. Chung, "A Nonparametric Probability Distribution Model for Short-Term Wind Power Prediction Error," in 2018 IEEE Canadian Conference on Electrical \& Computer Engineering (CCECE), 2018, pp. $1-5$.
20. Abdoli M.A., Golzary A., Hosseini A., Sadeghi P. Pellet Production Variables. In: Wood Pellet as a Renewable Source of Energy. University of Tehran Science and Humanities Series. Springer, Cham, 2018.

21. H. Sangrody, N. Zhou, S. Tutun, B. Khorramdel, M. Motalleb, and M. Sarailoo, "Long term forecasting using machine learning methods," in 2018 IEEE Power and Energy Conference at Illinois (PECI), 2018, pp. $1-5$

22. Abdoli M.A., Golzary A., Hosseini A., Sadeghi P. Biomass Densification. In: Wood Pellet as a Renewable Source of Energy. University of Tehran Science and Humanities Series. Springer, Cham, 2018. 